\title{
A Robot on the Operator's Chair - The Fine Line Between Automated Routine Operations and Situational Awareness
}

\author{
Jan-Christoph Scharringhausen*, Andreas Kolbeck ${ }^{\dagger}$, and Thorsten Beck ${ }^{\ddagger}$ \\ Deutsches Zentrum für Luft- und Raumfahrt e.V. (German Aerospace Center), \\ Münchener Str. 20, 82234 Wessling, Germany
}

\begin{abstract}
The European Data Relay System (EDRS) will provide a high speed data link between ground stations and satellites in low earth orbit. Up to 400 links per day are foreseen to be commanded by the groundsystems established at DLR's German Space Operations Center. The high command load is beyond the capabilities of a classical operational concept with manual operations. Therefore an automated system is established at the Devolved Payload Control Center with human interaction only necessary after a contingency, either in the ground processing or the space segment. One of the biggest challenges in this operational concept will be to become aware of the complex situation after any not-nominal situation. A reporting system will inform the on-call personnel of the current state of all components. This paper presents the operational concept established at the DPCC.
\end{abstract}

\section{Introduction}

Since decades robots are taking over manual work from us human beings. In most cases this puts humans into the position of an operator controlling the automated processes performed by the robots. In spacecraft operations the operator controls the systems on ground, like monitoring and control systems, which under his command operates the spacecraft. Usually no tasks are performed by the ground system without the command of the operator. On the other side the spacecraft provides the ground system with its telemetry which is displayed to the operator. It is his decision whether the presented values are as expected for the currently performed tasks or not. But what if the load of tasks is overwhelming for one human? What if too many tasks have to be performed to be observed by one operator?

The European Data Relay System (EDRS) will provide a high speed data link between ground stations and satellites in low earth orbit. On the first geostationary payload, EDRS-A, a laser communication terminal (LCT) as well as a Ka-band inter satellite antenna are available for the service. While a low speed $600 \mathrm{Mbit} / \mathrm{s}$ LCT link is possible in parallel to a Ka-band link, the high speed LCT link with $1800 \mathrm{Mbit} / \mathrm{s}$ can only be established while no Ka-band link is ongoing. The system is designed to perform up to 400 links per day, 200 with the optical channel and 200 via Ka-band.

Such a high command load combined with a required on-board autonomy of eight hours and a capability for late requests is beyond the capabilities of manual operational concepts. Only with the assistance of automated systems these high demands can be economically fulfilled. For the Devolved Payload Control Center (DPCC) established at DLR's German Space Operations Center (GSOC) an automatic command

*EDRS-A deputy flight director, German Space Operations Center, jan-christoph.scharringhausen@dlr.de.

$\dagger$ EDRS-A flight director, German Space Operations Center, andreas.kolbeck@dlr.de.

${ }_{\ddagger}^{\ddagger}$ EDRS-A ground system engineer, German Space Operations Center, thorsten.beck@dlr.de. 
and control system to operate the EDRS-A payload in its routine operations phase was developed. This innovative system will command the payload during nominal conditions entirely without human interaction. A Link Management System will trigger the flight procedures to be executed while an Automator will execute these procedures. In the flight procedures the steps are defined relative to a reference time. The Automator sends the telecommands with an execution time up to eight hours in the future to the spacecraft while the telemetry checks are queued and executed at the scheduled time. The Automator also represents a first step towards a procedure based commanding at the GSOC.

The human operator of a classical operations concept is replaced by an automatic system taking over for all nominal situations. Human interaction to this operations process is only necessary if a contingency occurs either in the processing on ground or at the payload in space. One of the biggest challenges in this operational concept will be to become aware of the complex situation after any not nominal situation. A reporting system will inform the on-call personnel of the current state of all components. According to this information he must bring the complete system into a safe state before any recovery action can be started. After the recovery, either on ground or in the space component, the nominal operations must be re-initiated from the safe state.

In the first sections the overall EDRS system with all its components on ground and in space will be presented followed by a deeper view into the DPCC, its role in the system, and its components. Afterwards the operational concept of the DPCC will be presented including the automated processes for the nominal routine operations and the manual interactions still necessary in nominal operations and during not nominal situations. Finally an assessment of the current situation and the way forward will be done.

\section{The European Data Relay System}

The European Data Relay System is designed to reduce time delays in the transmission and to increase the amount of data from low earth orbit satellites to the user. It is composed of two geostationary satellites positioned over Europe for in-orbit redundancy. The first payload (EDRS-A) is realized as a hosted payload on Eutelsat's EB9B satellite and has been launched in January 2016. The second (EDRS-C) will be a dedicated spacecraft based on the SmallGEO platform by OHB. Both satellites are equipped with a LCT to provide high speed optical links of up to $1800 \mathrm{Mbit} / \mathrm{s}$. In addition the EDRS-A satellite carries a Ka-band inter satellite antenna to establish links to LEO satellites. Both link types are using common RF equipment to relay the received data to the ground via Ka-band. ${ }^{1}$

The central part of the EDRS ground segment shown in figure 1 is the Mission Operations Center (MOC) established by Airbus Defence and Space. It interfaces with all other components and coordinates the overall mission. On the one hand it receives the link orders from the different users. It schedules the mission timeline for both EDRS satellites taken all known constraints into account and sends requests for the scheduled links to the Devolved Payload Control Center, operating the EDRS-A payload, and the Spacecraft Control Center (SCC), operating the EDRS-C satellite. It also coordinates with four groundstations based at Harwell, Weilheim, and Redu which receive the user data from both EDRS satellites and deliver the data to the users.

The DPCC is responsible for operating the EDRS-A hosted payload. A file based interface with Eutelsat's SCC which is operating the EB9B satellite is used for commanding. The telecommands (TC) of typically one flight operations procedure (FOP) are combined into one TC set file, transferred to the SCC in Paris, and released within Eutelsat's commanding system. For monitoring, two separated telemetry streams are received within the DPCC. One forwarded by the Eutelsat SCC including all satellite telemetry and one received at an EDRS dedicated antenna containing only LCT related data.

The MOC is connected to the DPCC via a second file based interface. The following request types are defined to be delivered by the MOC:

\section{Payload link configuration request}

The request type for each link execution either by the LCT or the Ka-band inter satellite antenna. It 


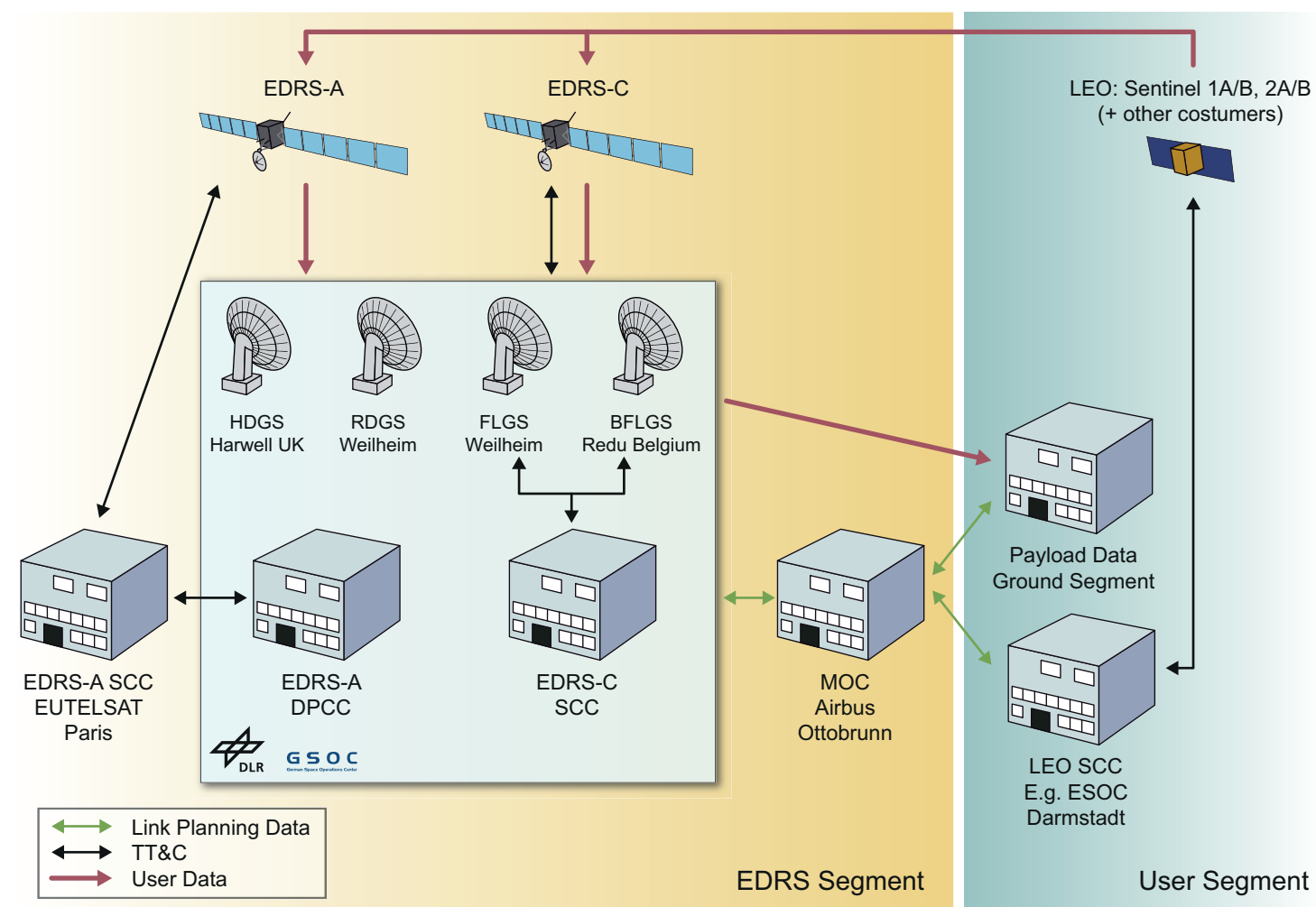

Figure 1: EDRS system overview

contains information of the link direction (EDRS to LEO, LEO to EDRS, or bidirectional), link speed, and LCT configuration parameters. It also contains coefficients of the LEO satellite's trajectory in the case of an LCT link, or vector data for the pointing direction of the Ka-band antenna.

\section{Payload routine configuration request}

A generic request type triggering the automatic execution of a flight operations procedure. The request contains the ID of the FOP, the necessary parameters for the FOP, and an execution time. Only a small number of FOP are possible to be requested for automated execution.

\section{Payload basic configuration request}

A generic request for the manual execution of a FOP. Similar to the routine request it contains the ID of the requested FOP, its parameters, and execution time. Contrary to a routine request this FOP is executed manually by the DPCC's flight operations team. Only a small quantity of requests of this type are expected and therefore no automatic mechanism is established.

\section{Forward tasking data request}

The LCT link service provides a mechanism to forward data from the EDRS GEO satellite to the target LEO satellite. These binary data are delivered to the DPCC via forward tasking data request and uploaded into the data processing unit (DPU) on-board EDRS.

\section{Payload configuration deletion request}

All request types above can be deleted by this request type. In the case that the DPCC's internal processing is ongoing the process is stopped internally. Otherwise, if the request is already processed into TC sets forwarded to the Eutelsat SCC, a FOP to delete the commands from the on-board time-tag TC buffer is generated and executed.

The DPCC is located inside the multi-mission environment of the GSOC. The very concept of multimission is based on the sharing of existing infrastructure (buildings, network, and software) with other missions. Within this environment a layered architecture of several components providing the EDRS service 
has been created. ${ }^{2}$ A Link Management System is processing the automatic requests received from the MOC. ${ }^{3}$ It interacts with a flight dynamics system and the Automator. The latter is the central component executing the automatized flight operations procedures. All components are reporting their current status to a common monitoring tool.

\section{DPCC operations}

In principal the operational concept for commanding and monitoring the EDRS-A payload is automatic. The sheer number of up to 400 links per day are the main driver for such an approach. An additional driver is the required speed of processing from receiving link requests from the MOC until sending commands to the spacecraft. A late request is possible until 45 minutes (reception at DPCC) before its execution. With the involved complexity of each link the processing is beyond the capability of manual commanding concepts.

\section{A. Flight Operations Procedures}

All operational tasks are based on flight operations procedures (FOP) defining the sequence of commands, telemetry verifications, and timings for a single task, such as the execution of a LCT link. With the FOP being executed by an automatic system the significance of the timing is even higher than for manual execution. Due to activities at the Eutelsat SCC, a delay of up to 15 minutes is possible between the processing of a command in the DPCC and the reception on-board the satellite. Additionally most telemetry values are transmitted once per 32 seconds by the frame based satellite which is based on the Airbus Eurostar 3000 platform. With some additional telemetry transmission and processing delay the verification of a telecommand, from its transmission by the DPCC until its execution confirmation in telemetry, might be more than 16 minutes. This delay must be taken into account at FOP development in order to be considered by the Automator.

To improve the functionality and reduce the number of FOP a limited amount of logical operations has been included in the design. Based on an input parameter the Automator can decide between different branches in the FOP. With this approach a single FOP can be used for similar tasks, regardless of the redundancy applied on the spacecraft. For example two identical parts of the data processing unit are present in the payload of EDRS-A. One part is active while the other one is available as cold redundancy in the case of a failure. Without branching two separate FOP would have been needed for each task using the DPU in order to handle the redundancy. With the branching possibility the currently used DPU is saved in a configuration file or database, and inserted into the input parameter of the FOP.

\section{B. Automated operations}

\section{Link operations}

The LMS is responsible for the planning of all link requests transmitted from the MOC. Usual these requests are available in the DPCC latest eight hours prior to their planned execution time as shown by the autonomy displayed in figure 2. In addition late requests are possible until 45 minutes before execution. At each LMS planning cycle, it compares the list of pending (not yet uplinked) link requests with the table mirroring the current state of the on-board time-tagged telecommand (TTC) buffer. As a result of this comparison, the LMS selects which activity (LCT link, Ka-band link, routine) shall be uplinked next. The LMS will always take the next activity in the chain (chronological sorted) and try to load it into the TTC buffer. In order to assess how many TTC slots are required for an activity, the LMS must know the number of TTC required by each FOP. The LMS scheduling process is triggered either after reception of a payload link configuration request or when the Automator delivers the current state of the TTC buffer to the LMS. This ensures low-latency LMS operation. In case not enough TTC slots are available for a pending complete activity, the LMS will postpone the uplink until the next planning cycle. Knowing the feedback from the Automator about the TTC buffer state, the LMS only uses the TTC buffer information that is verified by 
telemetry. Consequently it is ensured that enough space is really available in the TTC buffer before actually sending commands. As such, the TTC buffer on-board will be constantly kept as full as possible. Through these successive planning cycles, the LMS tries to schedule any request in its request database.

While the link planning process of an optical inter satellite link only requires the execution of one FOP, the process for Ka-band link is more complex. While one flight procedure is executed to configure the RF components of the payload for the link, the steering of the inter-satellite antenna is a complex task. The antenna is commanded by entries of a time and a direction embedded into two tables with 1440 entries each. The on-board function interpolates between two entries of the active table while new entries can only be commanded into the inactive one. The processing is started at a commanded start entry of one table. Certainly this entry must have a feasible time in the future and an antenna direction that is reachable in time without exceeding speed limits. For link requests received at the DPCC eight hours before their execution the table entries are written into the inactive table and a table switch is planned at a time when all the entries for the upcoming eight hours are in the new active table (figure 3a). For late requests or for the deletion of a link the currently running table would have to be changed. Therefore a new table for the next eight

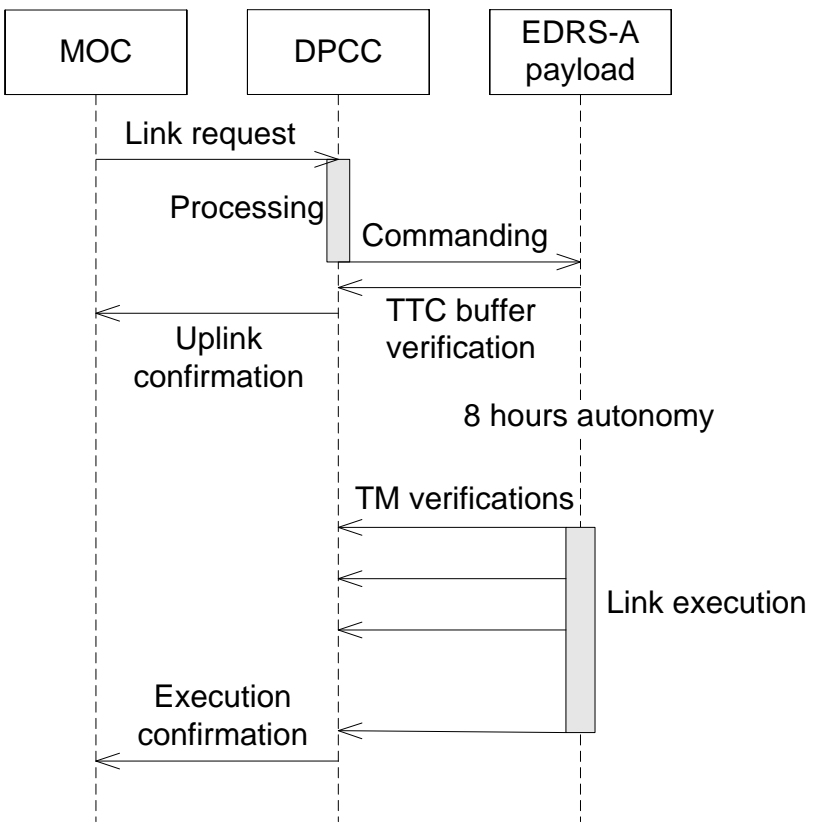

Figure 2: Optical link sequence hours including the late request (link $8 \mathrm{~b}$ in figure 3c), or excluding the deleted link (link 6 in figure 3b), is composed on ground and loaded to the inactive table on board. Afterwards the active table is switched to the new composed one.

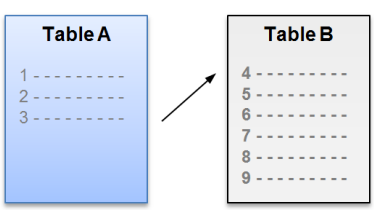

(a) Link addition

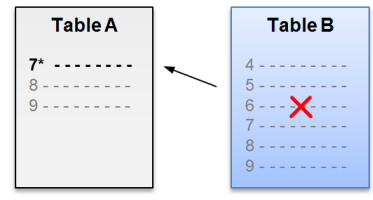

(b) Link deletion

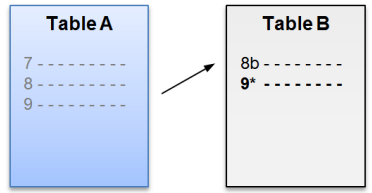

(c) Link insertion

Figure 3: Ka-table management: for each operation, the currently active table (in blue) must be switched

While the Ka-band link is always bidirectional, the LCT links are possible in return (from the target LEO satellite via EDRS to the ground), forward (to the LEO satellite), and bidirectional. The actual link commanding is similar in all three LCT link cases, but for forward and bidirectional links the binary data to be forwarded has to be uploaded to the DPU prior to link execution. This forward tasking data service is done via the normal commanding channel by telecommands containing 62 bytes of data. The filling of the DPU forward data buffer of five megabytes is planned by the LMS with a lower priority as the link commanding in order to not block the link execution.

\section{Routine operations}

In addition to the link operations a small number of routine operations are also performed automatically. These operations are also executed as time-tagged telecommands which are uploaded to the spacecraft eight hours in advance. Thereby the spacecraft autonomy of eight hours is ensured. Furthermore a synchronization between routine and link commands is needed. 
The following routine operations are conducted by the DPCC autonomously from request provided by the MOC:

\section{LCT time synchronization}

To perform the acquisition of a link with a counter terminal the LCT needs an accurate knowledge of the counter terminal's position. The target's trajectory is provided to the LCT via telecommand for each link. To calculate the actual position of the counter terminal the LCT depends on an accurate time reference. Therefor the LCT contains its own clock which is independent from the spacecraft clock and is adjusted to the GPS time reference. To perform the link acquisition within the specified time the LCT clock must be within a tolerance of $\pm 500 \mathrm{~ms}$. To ensure the required accuracy the LCT time is reported via telemetry every 2 seconds with an accuracy of $100 \mathrm{~ms}$. Within the DPCC the reported time is correlated to the GPS time taking the on-board processing time, the transmission time between the satellite and the groundstation, and the ground processing time into account. If this time correlation reveals a delta between the LCT on-board time and the GPS time of more than 250 msec, a time correction is scheduled by the LMS. This time correction is to be scheduled time-tagged to align it with the on-going link execution on board due to the fact that no time correction is possible during a link. At the given execution time the LCT clock is corrected by adding or subtracting the calculated milliseconds. This calculation also estimates and compensates for the drift.

\section{On-orbit propagator}

Besides the target position the LCT requires an accurate position of its own to calculate the line of sight to the counter terminal. The spacecrafts attitude data is provided by the platforms startracker while the orbit data has to be provided by ground. An on-orbit propagator is calculating the current orbit position based on orbit data calculated on ground. These OOP data are validate for a four hours time frame. Similar to the LCT time synchronization the OOP update must not be during an on-going link, therefore also the OOP upload is executed as time-tagged telecommand.

\section{LCT alignment matrix}

The LCT alignment must be calibrated to seasonal pointing biases. An accurate alignment matrix is required for a minimal uncertainty cone at link acquisition. The calibration is based on a comparison of the actual azimuth and elevation of the LCT during a link and the corresponding theoretical values. Based on these values calculated on ground and received via telemetry from the satellite, the DPCC flight dynamic system derives a corrected alignment matrix which is uploaded typically every 14 days. Usually the LCT is in an unparked position even between links and only parked during a station keeping maneuver, but to take the new alignment matrix into account the LCT must be in its park position. Therefore the alignment matrix upload is scheduled by the LMS during these maneuvers in order to not schedule an additional parking time which would reduce the services availability.

These routine operations are depending on data acquired from satellite telemetry or from DPCC flight dynamics. They have to be aligned with the scheduled link operations but are not needed as input for link scheduling. Therefore these tasks are prepared and scheduled within the DPCC without dependence to MOC requests. In addition the $\mathrm{MOC}$ requests routine operations via payload routine configuration request that are in dependence with the mission scheduling:

\section{LCT park and unpark}

Usually the LCT is kept in an unparked position in order to minimize the acquisition time of the links. Due to the risk of contamination of the LCT optic by particles, the LCT must be parked during platform thruster activation for station keeping maneuvers. Therefore a parking request is to be scheduled sufficient time before first thruster activation. Afterwards an unparking shall be scheduled to restore the nominal static configuration. These tasks are a mission scheduling responsibility and are therefore planned by the MOC and requested via routine requests to the DPCC.

\section{Ka-antenna steering pause}

To reduce attitude disturbances during a station keeping maneuver caused by a movement of the Kaband inter satellite antenna, the antenna steering shall be paused during the maneuver. To keep the 
antenna in a fix position the LMS inserts two entries into the steering table at the begin and the end of the requested pause, both with identical antenna positions. Also this is a mission scheduling responsibility and therefore planned on MOC side.

\section{Manual operations}

All other tasks are performed in a more classical manual operations approach. This is the case for all contingency operations as well as nominal operational tasks that are only performed rarely. For that members of the flight operations team will execute the FOP, and decide the timing and execution state. The telemetry verification can be done by display pages. But as the Automator is still used for generating the TC sets delivered to the Eutelsat SCC, a semi-automatic FOP execution is possible.

In the classical operations approach at GSOC the telecommands are loaded into a manual stack and executed one-by-one, or grouped together with a relative release time to each other. The execution of telecommands and telemetry validation is separated between the commanding system, providing the manual stack, and a display system providing the telemetry. The Automator provides the first step from this telecommand based commanding towards a procedure based commanding. Flight operations procedures are usually built using steps and sub-steps to generate a specific sequence. For the automatic execution presented in section B the Automator executes the complete FOP at once, meaning all telecommands are sent in one TC set to the SCC, where they are released one after the other with one second interval, and all TM verification are scheduled inside the Automator with a start time and a duration defined in the FOP. For manual operations this approach is not desired because it would remove the possibility of verifying the execution state by the operator. Therefore the manual execution is based on a step-by-step execution of the procedure. Now, the operator does not execute anymore one or a group of telecommands and verifies the telemetry in a separate system. He executes one step of the procedure, including the telecommands, the telemetry verification, and the timing of these. The Automator transmits the TC and verifies the TM, and reports the success or fail of the step back to the operator.

Manual flight operations procedures are requested via payload basic configuration requests from the MOC. While the LMS is taking care of the reporting to the MOC for automatic requests as shown in figure 2 , the reporting for basic requests must be done manually as well. This is realized by a web based tool guiding through the execution of the request. It displays the received request including the FOP ID, the necessary parameters, and the requested execution time. The DPCC team will then define a suitable time when the multi-mission schedule will allow to prepare and execute the request. During executing the FOP, the operator acknowledges the uplink and later the execution success within this webtool.

\section{Situational Awareness}

Being aware of the situation of all parts of the system is of the outmost significance of any spacecraft mission. Already in the classical operations concept the spacecraft controller (SPACON) is monitoring the spacecraft's status in telemetry as well as the state of the ground components. The added complexity is caused by the reduced knowledge of the ongoing activities triggered by the automatic system. The operator is not the actor anymore, but an observer. And with EDRS being included in the GSOC multi-mission environment the SPACON cannot monitor only EDRS at all times.

This new role reduces the situational awareness significantly. Mica Endsley defined the situation awareness (SA) as "the perception of the elements in the environment within a volume of time and space, the comprehension of their meaning and the projection of their status in the near future". ${ }^{4}$ Although the work of Mica Endsley is focused on aviation, the principles can be transferred to spacecraft operations. Three levels of SA have been defined in her work: ${ }^{5}$ Level 1 - Perception of the elements in the environment, Level 2 - Comprehension of the current situation, and Level 3 - Projection of the future status. Adapted to the operational scenario the three levels can be understood as: 
Level 1 Perceive the presence of a not nominal situation

Level 2 Comprehension of the system's situation and the processes going

Level 3 Projection of the future status

For the first level a simple and clear alarm mechanism must be installed. At the DPCC this is achieved by an acoustical alarm the SPACON is able to hear at all of his areas. While this mechanism is quite simple, the triggering of the alarm is only at a first glance. The first and most important step is to ensure that all components will communicate all their possible not nominal incidents. This includes not only failures within the component but also incidents of other components the component can get aware of. To ensure that this is completely covered is an easily underestimated task. Not only must all possible incidents be reported, it shall also be avoided to trigger the alarm for false positives. An alarm startling the operator every few seconds like the boy who cried wolf is easy to be not getting any attention anymore after a short while and might cover other alarms that would need immediate attention. For the spacecraft the alarm definition is done in the spacecraft reference database. It is possible to define alarms of different severity for all telemetry values generated by the satellite. These definitions are processed by the monitoring and control system SCOS, which is also generating the spacecraft related acoustical alarms. All ground components, like the LMS or also the ground hardware, are monitored by a GSOC built monitoring tool called NEMO. Originally build to monitor network components the tool was adapted to monitor the DPCC system. All components deliver their status to NEMO which generates an acoustical alarm to alert the SPACON. ${ }^{2}$

In level 2 an understanding of the situation must be achieved. While this is inevitable to solve the not nominal situation, it is considered not as critical as level 1 . In case level 1 awareness cannot be obtained, a possibly the mission endangering incident might be missed. For level 2 three different stages are foreseen within the DPCC's operations. At first level the spacecraft controller is gathering information on the situation. The tool NEMO is presenting him synoptic displays to recognize the affected component in the network (figure 4a) or the software components (figure 4b). For alarms generated by the spacecraft's telemetry the tool SATMON used at GSOC is used to provide the TM values on display pages as well as graphical plots (figure 4d). With this information the SPACON is guided by a ground operations procedure (GOP) to act accordingly. For all previously known and easy to resolve not nominal situation the GOP is leading the SPACON to a solution to be implemented by him. In any other case the next level must be activated by informing on-call GSOC engineers. The engineers with a deeper knowledge of the systems will use additional information like logfiles to gather an understanding of the on-going situation.

For level 3 the operators need an understanding not only on the happened incident but also on the still on-going operations. The most important tool is a display of the scheduled tasks of the mission. A timeline display as shown in figure $4 \mathrm{c}$ is used to be aware of this aspect. With this information it is possible to assess in what way the mission might be affected by the incident and at what point in time a corrective action should be conducted. First priority over the on-going operations is granted to the safety of the components, especially on board the spacecraft. Therefore mechanism to stop the operations and bringing all components into a safe state are implemented.

\section{Conclusion}

The EDRS system is setting new speed levels, not only for data transmission from space but also in the operations for GEO satellites. The innovative automated operations at DPCC are now starting to be in use for the first time and handle this challenging mission after the manual in-orbit test campaign of EDRS-A is completed. After the DPCC systems will have shown its capability in automatic operations, the next improvements will be assessed for EDRS-C. Additional routine operations, such as station keeping maneuvers, might be able to be automatized with this cutting edge concepts. 


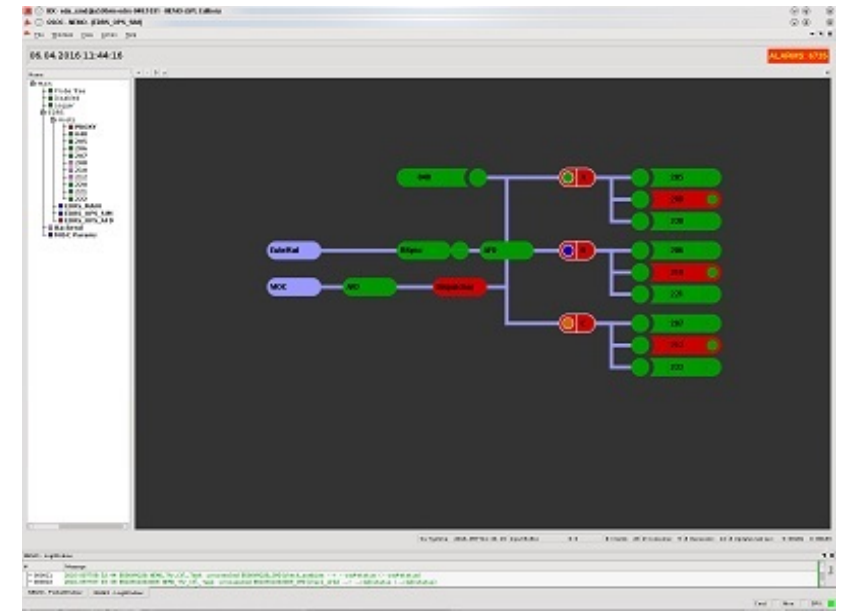

(a) Network monitoring

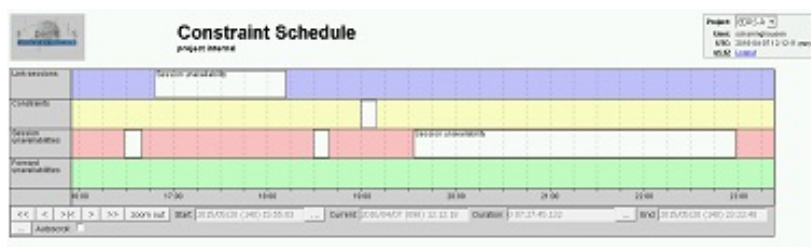

(c) Timeline monitoring

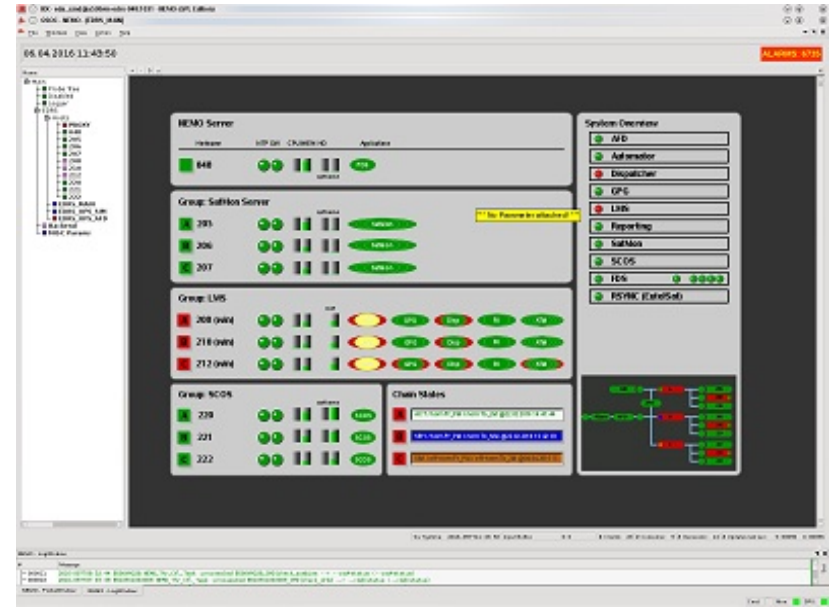

(b) Component monitoring

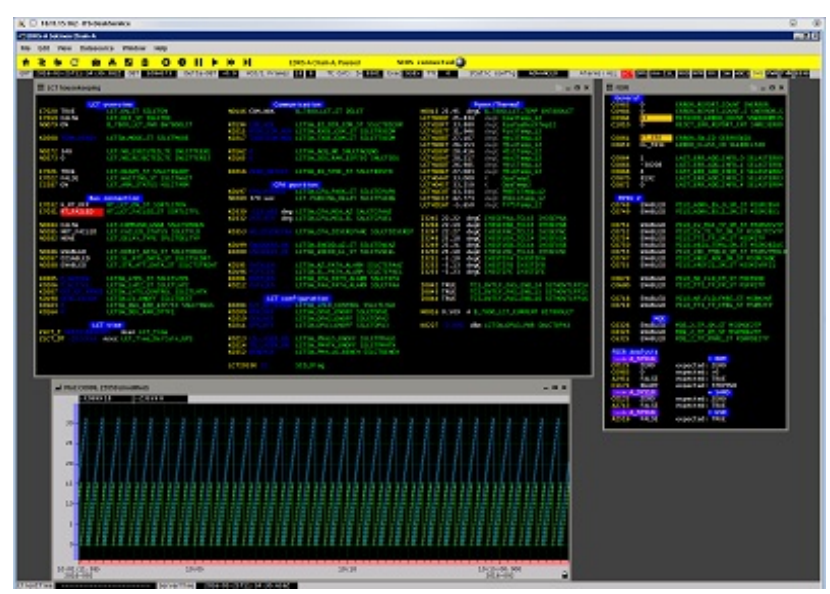

(d) Telemetry monitoring

Figure 4: Monitoring screens of different situation aspects 


\section{Nomenclature}

DLR Deutsches Zentrum für Luft- und Raumfahrt e.V. (German Aerospace Center)

DPCC Devolved Payload Control Center

DPU Data Processing Unit

EDRS European Data Relay System

FOP Flight Operations Procedure

GOP Ground Operations Procedure

GSOC German Space Operations Center

LCT Laser Communication Terminal

LMS Link Management System

MOC Mission Operations Center

OOP On-orbit propagator

RF Radio Frequency

SA Situational Awareness

SCC Spacecraft Control Center

SPACON Spacecraft Controller

TC Telecommand

TTC Time-Tagged Telecommand

\section{References}

\footnotetext{
${ }^{1}$ Wallrapp, F., Ballweg, R., and Gataullin, Y., The European Data Relay System (EDRS): Operational Challenges, 62nd International Astronautical Congress, 03-07 October 2011, Cape Town, South Africa.

${ }^{2}$ Beck, T., Schmidhuber, M., and Scharringhausen, J., Automation of Complex Operational Scenarios - Providing $24 / 7$ Inter-Satellite Links with EDRS, 14th International Conference on Space Operations, 16-20 May 2016, Daejon, Korea (submitted for publication).

${ }^{3}$ Göttfert, T., Grishechkin, B., Wörle, M., and Lenzen, C., The Link Management System for the European Data Relay System, 14th International Conference on Space Operations, 16-20 May 2016, Daejon, Korea (submitted for publication).

${ }^{4}$ Endsley, M., Design and evaluation for situation awareness enhancement, Proceeding of the Human Factors Society 32nd Annual Meeting, Santa Monica, CA: Human Factors Society, 1988, pp. 97-101.

${ }^{5}$ Endsley, M., Situation Awareness, The Oxford handbook of cognitive engineering, Oxford Univ. Press, New York, 2013, pp. 88-108.
}

10 of 10 